\title{
Changes in biometry and cerebroplacental hemodynamics in fetuses with congenital heart diseases
}

\author{
Alberto Borges Peixoto ${ }^{1,2}$, Gabriele Tonni ${ }^{3}$, Edward Araujo Júnior ${ }^{2}$ \\ ${ }^{1}$ Mario Palmério University Hospital - University of Uberaba (UNIUBE), Uberaba-MG, Brazil; ${ }^{2}$ Department of Obstetrics, Paulista School \\ of Medicine - Federal University of São Paulo (EPM-UNIFESP), São Paulo-SP, Brazil; ${ }^{3}$ Department of Obstetrics and Gynecology, Prenatal \\ Diagnostic Center, Guastalla Civil Hospital, Reggio Emilia, Italy \\ Correspondence to: Prof. Edward Araujo Júnior, PhD. Rua Belchior de Azevedo, 156 apto, 111 Torre Vitoria, São Paulo-SP, CEP 05089-030, Brazil. \\ Email: araujojred@terra.com.br.
}

Submitted Sep 08, 2016. Accepted for publication Sep 12, 2016.

doi: $10.21037 /$ jtd.2016.10.71

View this article at: http://dx.doi.org/10.21037/jtd.2016.10.71

Multiple factors can influence fetal blood flow, including placental function, heart anatomy and distal vascular bed impedance (1). Nutrients and oxygen undergo exchange between fetal and maternal circulation at the level of placental villi. Placenta extracts a fixed proportion of the nutrient stream ( $70 \%$ of glucose and $40 \%$ of oxygen supplied to the uterus) whereas fetal nutrition is restricted to the surplus that remains after placental demands (2).

There are three shunts pathways in fetal circulation: ductus venosus (DV), foramen ovale (FO) and ductus arteriosus (DA). They are responsible to distribute oxygenated and deoxygenated blood supply to the placenta and fetal organs (3). Oxygenated blood from placenta is directed toward the fetus into the umbilical vein (UV). Atrial septum and Eustachian valve works together to direct oxygenated blood from DV across the FO to the left atrium (3). The deoxygenated blood from the superior vena cava (SVC) and inferior vena cava (IVC) are directed to the right atrium. During diastole, the oxygenated and deoxygenated bloods are directed to the left and right ventricle, respectively. During systole, the oxygenated blood from the left ventricle is directed to the myocardium, head, upper and lower body. Opposite, the deoxygenated blood from the right ventricle is directed to the placenta across the main pulmonary artery and DA (3). The right ventricular output is greater than the left output, although the difference is not substantial (3).

With diminished oxygen content, there is a fetal protective mechanism, termed brain sparing, that decrease the cerebral vascular resistance and results in an increase in cerebral flow $(4,5)$. During this adaptive response, Doppler ultrasound evaluation might detect a decrease pulsatility index (PI) in the middle cerebral artery (MCA), a decrease in cerebral/placental ratio (CPR) and an increased UA PI. MCA PI below 5th centile for gestational age, may be found in fetuses with growth restriction, can predict poor neurologic outcome besides being associated with higher mortality rate $(4,5)$.

It is well established that fetal cardiovascular manifestation of placental dysfunction is different for those fetuses that developed early onset fetal growth restriction (FGR) (occuring before 32 weeks) and late onset FGR (occuring after 32 weeks) (6). In early-onset FGR, late cardiovascular manifestations of placental dysfunction become more likely when the end-diastolic velocity UA is reversed (REDV-UA) (7). The typical pattern of deterioration progresses from escalating abnormalities in UA and venous Doppler parameters to abnormal biophysical parameters (7-10).

With increasing placental dysfunction, there is a decrease of glucose transfer to the heart and brain; lactate and ketones are metabolized as primary energy sources (11). The impairment and deficiency of nutrients supply has been linked independently to a wide range of neurodevelopmental disorders (2).

In contrast, there is a less severe placental dysfunction in late onset FGR, with cardiovascular modifications not extending beyond cerebral circulation. In these fetuses, Doppler ultrasound evaluation have shown decreased CPR, with either normal or slightly elevated UA PI (12). 
Although FGR at term, does not present with the same degree of clinical deterioration as does early-onset FGR, abnormal brain microstructure and metabolism have been documented independently of the degree of Doppler waveforms abnormalities (13).

In fetuses with early-onset FGR, the risk of neurological sequelae, motor and cognitive delay, increases as REDVUA decreases. The UA waveforms reflect the severity of placental dysfunction and affect child neurodevelopment independently in case of early-onset FGR. In lateonset FGR, abnormal UA Doppler waveforms are a less prominent feature and, developmental abnormalities ensue in other districts that appear to be related to specific brain areas and higher brain functions (6).

In contrast, MCA Doppler waveforms in early-onset FGR provide little additional information over UA Doppler alone. In late-onset FGR, the MCA Doppler provides important information regarding blood flow resistance and pattern of development of different areas of the fetal brain. In the neonate, this results as decrease motor performance, social-interactive and attention deficits, while in infancy and early childhood, performance attention, communication, problem solving, emotion and social function may be affected $(6,14)$.

Congenital heart disease (CHD) affects up to $1 \%$ of all live births and is a leading cause of neonatal mortality and morbidity (13). CHD is considered a risk factor for FGR, even in the absence of associated chromosomal abnormalities $(15,16)$. However, the underlying mechanism has not yet been clearly elucidated. Explanations have been put forward to explain the association between CHD and FGR. Embryos with intrinsic growth disturbances are at an increased risk of developmental abnormalities during cardiogenesis. Other explanation may be that fetal circulatory patterns in the presence of specific cardiovascular malformations are incompatible with optimal fetal growth [16]. Recently, it has been reported that fetuses with CHD have decreased placental growth factor (PlGF) at 11-13 weeks' gestation (17). Furthermore, theses fetuses have higher anti-angiogenic expression characterised by soluble fms-like tyrosine kinase-1 (sFlt-1), vascular endothelial growth factor A (VEGF-A) and soluble endoglin (sEng) than controls (18). Theses results might suggest that placental impairment contributes to impair the potential growth of fetuses with CHD.

Ballweg et al. (19) have shown that fetuses with CHD exhibits poor neurologic development primary caused by cardiac surgical interventions. However, there is evidence that that brain injuries in CHD patients may be detected at birth and may be caused by chronic cerebral hypoxia due to direct delivery of deoxygenated blood or by intracardiac mixing of oxygenated and deoxygenated blood during the fetal period (20).

Donofrio et al. (20) were the first to report reduced head circumference (HC) measurements in fetuses with CHDs in the third trimester. Similarly, Arduini et al. (21) found lower HC at 34 weeks' gestation. Masoller et al. (22) documented, for the first time, that a remarkable fraction of fetuses with CHD already have biparietal diameter (BPD) and HC values below the 5 th percentile (50\% and $25 \%$, respectively) for expected gestational age in the second trimester of pregnancy. Opposite, in fetuses with FGR due placental insufficiency occuring during mid-gestation, the abdominal circumference (AC) and femur length (FL) are more growth restricted than $\mathrm{HC}$ values (6).

During recent years, CHD has been diagnosed earlier due to technical advancement of the ultrasound equipment, especially with the widespread use of high-definition realtime apparatus. Prenatal counseling of CHD is challenging for fetal medicine specialists although the extended knowledge of these diseases together with cardiac surgery advancements are still related to critical survival rates and poor neurodevelopment later in life $(23,24)$.

Masoller et al. (25) performed a study with 58 fetuses with CHDs vs. 58 controls using Doppler ultrasound, head biometry and magnetic resonance imaging (MRI), respectively between 20-24 weeks and 36-38 weeks' gestation. In this study, fetuses with CHD had significantly smaller mean Z-scores of BPD and HC, and a higher proportion of fetuses with $\mathrm{BPD}$ and $\mathrm{HC}<5$ th centile. No differences in AC and FL were observed. Fetuses with CHDs showed significantly lower mean Z-scores for MCA-PI and CPR than controls. Regarding the MRI results, fetuses with CHDs have shown significantly smaller brain, intracranial and opercular volumes than did controls. Furthermore, fetuses with $\mathrm{CDH}$ have presented significantly decreased depths for left and right parietoccipital, cingulate and calcarine fissures and, significantly increased depth of the left insular lobe compared to controls. Regarding spectroscopic analysis, fetuses with CHD haves shown an increased Ino (myoinositol)/(choline) Cho ratios in the frontal lobe and basal ganglia and decreased NAA (N-acetylaspartate)/Cho and $\mathrm{Cho/(creatine)} \mathrm{Cr}$ ratios in both areas compared to controls. Newborns not requiring open-heart surgery during the first 6 months of age, the Bayley Scales of Infant and Toddler 
Development, third edition (BSID-III) (26) was carried out to evaluate the neurodevelopmental outcome. The neurodevelopment outcome reported at moths/years results have found linear correlations between average BSID-III scores and total brain volume $(r=0.410 ; \mathrm{P}=0.02)$, left and right cingulate fissure depth $(\mathrm{r}=0.359 ; \mathrm{P}=0.04$ and $\mathrm{r}=0.337$; $\mathrm{P}=0.04$, respectively), frontal Ino/Cho ratio $(\mathrm{r}=-0.531$; $\mathrm{P}<0.01)$ and NAA/Cho ratio $(\mathrm{r}=0.452 ; \mathrm{P}<0.01)$.

Ruiz et al. (27) conducted an interesting retrospective study in two tertiary care centers in Spain, where 444 ultrasound examinations were performed between 20-24 weeks' gestation and 119 fetuses were detected with CHD. The primary aim of this study was to ascertain whether impairment in fetal head and body growth develops throughout gestation whereas the secondary aims were to establish if changes occurring could be related to the particular type of oxygen delivery to fetal brain and whether placental perfusion could be related to changes in head and body size in fetuses with CHD.

In order to analyze the effect of cerebral haemodynamics, according to the expected main pattern of placental (oxygenated, nutrient-rich) vs. systemic (deoxygenated, nutrient-poor) mix of blood supply to the fetal brain in different type of CHD, the study population was stratified as follows, using a previously published classification (22):

(I) Group 1: expected low placental blood content, including severe left outflow tract obstruction with reversed flow in the aortic isthmus (and thus retrograde cerebral blood perfusion from the DA), and transposition of the great arteries (TGA) with the aortic blood flow originating from the right ventricle.

(II) Group 2: expected intermediate placental and systemic blood content owing to intracardiac shunts (septal defects, conotruncal defects other than TGA and complex CHD).

(III) Group 3: expected high placental blood content, in which there was brain perfusion originated from the left ventricle via a correctly formed aorta (right CHD), including pulmonary stenosis, tricuspid atresia, Ebstein's anomaly and pulmonary atresia.

Ruiz et al. (27) have found that fetuses with CHDs presented a small head at ultrasound diagnosis (BPD $-1.32 \pm 0.99 \mathrm{Zs}, \mathrm{HC}-0.79 \pm 1.02 \mathrm{Zs}$ ), which remained small throughout gestation, a finding that was confirmed at birth (HC Zs $-0.56 \pm 1.21,71^{\text {st }}$ centile). Fetuses with $\mathrm{CDH}$ had normal AC $(0.49 \pm 0.88 \mathrm{Zs}$, 69th centile) and FL $\left(-0.04 \pm 0.49 \mathrm{Zs}, 48^{\text {th }}\right.$ centile $)$ measurements. Doppler ultrasound waveforms analyses were within normal ranges in fetuses with CHDs in the second trimester: UA-PI Zs $\left(-0.45 \pm 0.65,33^{\text {rd }}\right.$ centile $)$, MCA-PI Zs $\left(-0.36 \pm 1.28,36^{\text {th }}\right.$ centile), CPR Zs $\left(-0.29 \pm 1.37,39^{\text {th }}\right.$ centile) and UtA-PI Zs $\left(0.17 \pm 2.20,57^{\text {th }}\right.$ centile). However, $18 \%$ of fetuses with CHDs presented an MCA and CPR below the 5th centile at the time of first examination. UtA and UA Doppler ultrasound parameters showed a significant increase towards the end of pregnancy, whereas no significant changes were observed for MCA or CPR with advancing gestational age. Both MCA and CPR presented significant differences in longitudinal behavior between CHD groups.

The main observations of the study by Ruiz et al. (27) were that head biometry measurements remained smaller than normal throughout gestation and that placental hemodynamics showed impairment towards the end of pregnancy, regardless of the type of CHD anomaly. These findings might suggest that the cause of abnormal brain development could be already present from the beginning of pregnancy.

The identification of prenatal markers of abnormal neurodevelopment in fetuses with CHD might be useful to better understand the fetal pathophysiologic changes and improve clinical counseling.

The study by Ruiz et al. (27), although a retrospective analysis with small samples of fetuses with different $\mathrm{CHD}$, may contribute to increase our knowledge on the potential growth of fetuses with $\mathrm{CDH}$. In a previous study by the same authors (23), a linear correlation between neurodevelopment delay and clinical variables such as fetal brain volume, brain fissure depth and metabolic rations on MRI evaluation were demonstrated.

In summary, it is well established that placental dysfunction is related to fetal growth restriction. Doppler ultrasound evaluation is an important tool to better understand of hemodynamic adaptation process whose fetuses suffer and then predict neonatal neurodevelopment outcomes. CHD is a risk factor for FGR, however, the underlying mechanism has not yet been clearly elucidated. It is look like that in fetuses with CHD, there is decrease in angiogenic factors and increase in anti-angiogenic factors, predisposing placental dysfunction. Fetuses with CHD have a small head from the second trimester onwards that remain smaller than normal throughout pregnancy, and a significant trend towards increased resistance in the UtA and UA Doppler. These findings are related with neurodevelopment delay even prior open cardiac surgery. The correlation between CHD, placental dysfunction across the pregnancy, 
smaller fetal head, smaller fetal brain and neurodevelopment delay allows improving the counseling regarding the prediction and prevention of abnormal neurodevelopment in CHD cases.

\section{Acknowledgements}

None.

\section{Footnote}

Conflicts of Interest: The authors have no conflicts of interest to declare.

\section{References}

1. Heymann MA, Rudolph AM. Effects of congenital heart disease on fetal and neonatal circulations. Prog Cardiovasc Dis 1972;15:115-43.

2. Baschat AA. Fetal responses to placental insufficiency: an update. BJOG 2004;111:1031-41.

3. Rudolph AM. The fetal circulation and postnatal adaptation. In: Rudolph AM (ed) Congenital Diseases of the Heart: Clinical-Physiological Considerations, 2nd edn. New York 2001;3-43.

4. Mari G, Deter RL. Middle cerebral artery flow velocity waveforms in normal and small-for-gestational-age fetuses. Am J Obstet Gynecol 1992;166:1262-70.

5. Rizzo G, Arduini D, Luciano R, et al. Prenatal cerebral Doppler ultrasonography and neonatal neurologic outcome. J Ultrasound Med 1989;8:237-40.

6. Baschat AA. Neurodevelopment following fetal growth restriction and its relationship with antepartum parameters of placental dysfunction. Ultrasound Obstet Gynecol 2011;37:501-14.

7. Ferrazzi E, Bozzo M, Rigano S, et al. Temporal sequence of abnormal Doppler changes in the peripheral and central circulatory systems of the severely growth-restricted fetus. Ultrasound Obstet Gynecol 2002;19:140-6.

8. Baschat AA, Gembruch U, Harman CR. The sequence of changes in Doppler and biophysical parameters as severe fetal growth restriction worsens. Ultrasound Obstet Gynecol 2001;18:571-7.

9. Cosmi E, Ambrosini G, D'Antona D, et al. Doppler, cardiotocography, and biophysical profile changes in growth-restricted fetuses. Obstet Gynecol 2005;106:1240-5.

10. Vintzileos AM, Fleming AD, Scorza WE, et al.
Relationship between fetal biophysical activities and umbilical cord blood gas values. Am J Obstet Gynecol 1991;165:707-13.

11. Vannucci RC, Vannucci SJ. Glucose metabolism in the developing brain. Semin Perinatol 2000;24:107-15.

12. Turan OM, Turan S, Gungor S, et al. Progression of Doppler abnormalities in intrauterine growth restriction. Ultrasound Obstet Gynecol 2008;32:160-7.

13. Hoffman JI, Kaplan S. The incidence of congenital heart disease. J Am Coll Cardiol 2002;39:1890-900.

14. Oros D, Figueras F, Cruz-Martinez R, et al. Middle versus anterior cerebral artery Doppler for the prediction of perinatal outcome and neonatal neurobehavior in term small-for-gestational-age fetuses with normal umbilical artery Doppler. Ultrasound Obstet Gynecol 2010;35:456-61.

15. Perez-Delboy A, Simpson LL. Prenatal sonographic diagnosis of congenital heart disease and intrauterine growth restriction: a case-control study. J Clin Ultrasound 2007;35:376-81.

16. Rosenthal GL. Patterns of prenatal growth among infants with cardiovascular malformations: possible fetal hemodynamic effects. Am J Epidemiol 1996;143:505-13.

17. Llurba E, Syngelaki A, Sánchez O, et al. Maternal serum placental growth factor at 11-13 weeks' gestation and fetal cardiac defects. Ultrasound Obstet Gynecol 2013;42:169-74.

18. Llurba E, Sánchez O, Ferrer Q, et al. Maternal and foetal angiogenic imbalance in congenital heart defects. Eur Heart J 2014;35:701-7.

19. Ballweg JA, Wernovsky G, Gaynor JW. Neurodevelopmental outcomes following congenital heart surgery. Pediatr Cardiol 2007;28:126-33.

20. Donofrio MT, Bremer YA, Schieken RM, et al. Autoregulation of cerebral blood flow in fetuses with congenital heart disease: the brain sparing effect. Pediatr Cardiol 2003;24:436-43.

21. Arduini M, Rosati P, Caforio L, et al. Cerebral blood flow autoregulation and congenital heart disease: possible causes of abnormal prenatal neurologic development. J Matern Fetal Neonatal Med 2011;24:1208-11.

22. Masoller N, Martínez JM, Gómez O, et al. Evidence of second-trimester changes in head biometry and brain perfusion in fetuses with congenital heart disease. Ultrasound Obstet Gynecol 2014;44:182-7.

23. Donofrio MT, Massaro AN. Impact of congenital heart disease on brain development and neurodevelopmental outcome. Int J Pediatr 2010;2010. pii: 359390. 
24. Khalil A, Suff N, Thilaganathan B, et al. Brain abnormalities and neurodevelopmental delay in congenital heart disease: systematic review and meta-analysis. Ultrasound Obstet Gynecol 2014;43:14-24.

25. Masoller N, Sanz-CortéS M, Crispi F, et al. Midgestation brain Doppler and head biometry in fetuses with congenital heart disease predict abnormal brain development at birth. Ultrasound Obstet Gynecol

Cite this article as: Peixoto $\mathrm{AB}$, Tonni G, Araujo Júnior E. Changes in biometry and cerebroplacental hemodynamics in fetuses with congenital heart diseases. J Thorac Dis 2016;8(10):E1282-E1286. doi: 10.21037/jtd.2016.10.71
2016;47:65-73.

26. Bayley N. Bayley Scales of Infant and Todler Development (3rd edn). San Antonio: PsychCorp. 2006.

27. Ruiz A, Cruz-Lemini M, Masoller N, et al. Longitudinal changes in fetal biometries and cerebroplacental haemodynamics in fetuses with congenital heart disease. Ultrasound Obstet Gynecol 2016. [Epub ahead of print]. 